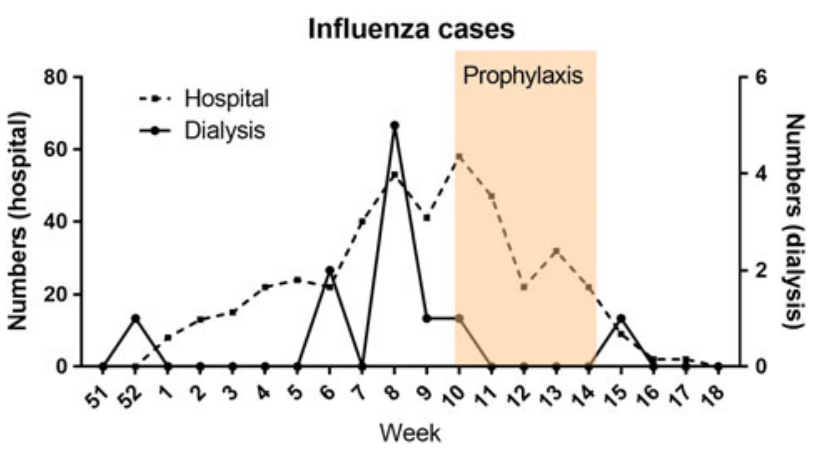

Fig. 1. The number of influenza cases per week is shown for the hospital (left axis) and the hemodialysis unit (right axis). The red box denotes the period the prophylaxis was administered to hemodialysis patients.

impaired immune response in hemodialysis patients to influenza vaccination, even after a booster vaccine dose, and its real effect on clinical outcomes and mortality is uncertain., ${ }^{2,3}$ Oseltamivir is a neuramidase inhibitor licensed for the treatment of and prophylaxis for influenza. It is mainly renally eliminated and is cleared by hemodialysis (molecular mass $312,4 \mathrm{~g} / \mathrm{mol}$ ). The optimal dose for prophylaxis in hemodialysis patients in unknown. A population pharmacokinetic study suggested $30 \mathrm{mg}$ every other hemodialysis session, ${ }^{4}$ whereas another clinical study on influenza A/H1N1 used $75 \mathrm{mg}$ every 5 days. ${ }^{5}$ These studies, however, have been performed in the era of low-flux filters, while hemodiafiltration (HDF) and highflux dialyzers are the current standard of care.

In the 2017-2018 influenza season, we started oseltamivir 30 mg after each hemodialysis session in our dialysis unit because of an emerging outbreak, even though all our patients had been vaccinated with a standard-dose quadrivalent vaccine. More precisely, 5 new infections occurred in week 8 (Figure 1). Additionally, the number of patients with influenza in our hospital, a 1,182-bed acute- and tertiary-care hospital, was rising sharply (Figure 1). In patients with influenza-like symptoms, testing for influenza $\mathrm{A}$ and $\mathrm{B}$ on combined nasopharyngeal-throat swabs was performed using real-time PCR on Taqman array cards. Of the 137 patients in our dialysis unit, 130 (mean age, $73.1 \pm 12 \mathrm{y}$; $36 \%$ female) provided informed consent to start the prophylactic treatment. Prophylaxis was administered from week 10 up to week 14 , given the dropping number of influenza cases in the hospital. Of these 130 patients, 7 (5\%) stopped prophylaxis early because of their own decision $(n=3)$ or reported adverse effects: stomachache $(\mathrm{n}=1)$ and muscle aches $(\mathrm{n}=3)$. During the period of prophylaxis, no case of influenza was detected in our dialysis unit (Figure 1). On average, the number of patients screened for influenza was 2.58 per week during the period of prophylaxis, and it had been 2.34 per week during the 11 previous weeks, indicating continued screening of patients with influenza-like symptoms. One week after the end of prophylaxis, 1 case of influenza occurred in our unit.

Our observational study shows it is feasible and safe to administer prophylaxis for influenza with oseltamivir in hemodialysis patients. To overcome potential enhanced elimination by high-flux dialysis and HDF, we chose $30 \mathrm{mg}$ after each hemodialysis session in contrast to dosing every other hemodialysis session, as indicated in the packet insert and as suggested by a population pharmacokinetic approach. ${ }^{4}$ Dosing after hemodialysis also allowed for optimal compliance. No major adverse effects were observed, and $95 \%$ of the patients continued the prophylaxis during the predetermined period. Although no control group was included, the absence of new influenza cases in our hemodialysis, despite the continuing intensive influenza epidemic in our hospital, indicates the effectiveness of this strategy.

In light of recent findings that vaccination remains a suboptimal strategy to prevent influenza in hemodialysis patients, ${ }^{2,3}$ we propose to administer oseltamivir prophylaxis during influenza season as an additional protective measure. Further studies should confirm the effectiveness of this strategy and explore its costeffectiveness.

\section{Acknowledgments. None.}

Financial support. No financial support was provided relevant to this article.

Conflicts of interest. All authors report no conflicts of interest relevant to this article.

\section{References}

1. Brankston G, Gitterman L, Hirji Z, Lemieux C, Gardam M. Transmission of influenza A in human beings. Lancet Infect Dis 2007;7:257-265.

2. Liao Z, Xu X, Liang Y, Xiong Y, Chen R, Ni J. Effect of a booster dose of influenza vaccine in patients with hemodialysis, peritoneal dialysis and renal transplant recipients: a systematic literature review and meta-analysis. Hum Vaccin Immunother 2016;12:2909-2915.

3. Remschmidt $\mathrm{C}$, Wichmann $\mathrm{O}$, Harder T. Influenza vaccination in patients with end-stage renal disease: systematic review and assessment of quality of evidence related to vaccine efficacy, effectiveness, and safety. BMC Med 2014;12:244.

4. Kamal MA, Lien KY, Robson R, et al. Investigating clinically adequate concentrations of oseltamivir carboxylate in end-stage renal disease patients undergoing hemodialysis using a population pharmacokinetic approach Antimicrob Agents Chemother 2015;59:6774-6781.

5. Choo D, Hossain M, Liew P, Chowdhury S, Tan J. Side effects of oseltamivir in end-stage renal failure patients. Nephrol Dial Transplant 2011;26: 2339-2344.

\title{
Fifty years of success in controlling tuberculosis in Iran, the question is how?
}

\author{
Vahid Lohrasbi PhD ${ }^{1}$, Neda Shirmohammadlou Msc ${ }^{2}$ and Davood Darban-Sarokhalil PhD ${ }^{1}$ \\ ${ }^{1}$ Department of Microbiology, Faculty of Medicine, Iran University of Medical Sciences, Tehran, Iran and ${ }^{2}$ Department of Microbiology, Faculty of Medicine, Zanjan \\ University of Medical Sciences, Zanjan, Iran
}

Author for correspondence: Davood Darban-Sarokhalil, Email: davood_darban@yahoo. com or darban.d@iums.ac.ir.
Cite this article: Lohrasbi V, et al. (2019). Fifty years of success in controlling tuberculosis in Iran, the question is how?. Infection Control \& Hospital Epidemiology, 40: 498-499, https:// doi.org/10.1017/ice.2019.30 
To the Editor-Tuberculosis (TB) is one of the top 10 causes of death worldwide. ${ }^{1}$ Despite efforts over the last two decades, control of the infection at a global level is still out of reach; however, decreasing trends in Mycobacterium tuberculosis incidence, outbreak, and mortality have been observed. ${ }^{2}$ The best estimate for 2017 is 1.3 million TB deaths (range, $1.2-1.4$ million) with an additional 300,000 deaths (range, 266,000-335,000) resulting from TB disease among HIV-positive people. ${ }^{3}$ Most cases are estimated to have occurred in Asia and Africa, with the highest incidence in China (1,410 million) and India (1,339 million) in 2017..$^{3}$ According to the data, ${ }^{3,4}$ Iran has a low TB mortality rate (1 case per 100,000 population, excluding cases of HIV plus TB), compared to most neighboring countries such as Afghanistan (29 per 100,000 population), Armenia (1.2 per 100,000 population), Azerbaijan (8.7 per 100,000 population), Iraq (2.9 per 100,000 population), Turkmenistan ( 9.9 per 100,000 population), and Pakistan (27 per 100,000 population). Moreover, according to a report by the Ministry of Health and Medical Education of Iran, from 1964 to 2016, the incidence of pulmonary TB with positive sputum smear in Iran decreased by $>10$ times to 5.7 per 100,000 population in $2016 .{ }^{5}$ But how has Iran succeeded in controlling TB in a region with high burden of tuberculosis and multidrug-resistant (MDR) tuberculosis?

Because Iran is located in a special geographical location, achieving this result requires huge financial cost, equipment, and time investment for Iran. The country is surrounded by 2 countries with a high burdens of TB (ie, Pakistan and Afghanistan) in the east as well as Iraq in the west (with which Iran is involved in external and internal wars). In addition, Azerbaijan to the north has a high prevalence of MDR-TB. Thus, the challenge to control this disease is substantial, and its success is admirable. There are several important reasons for Iran's success. (1) Continuation of free treatment and diagnosis for TB patients plays a significant role in TB control. (2) Nine regional TB laboratories in big cities including Tabriz, Shiraz, Isfahan, Mashhad, Kermanshah, Zahedan, Gorgan, Ahvaz, and Tehran distribute alerts about all TB incidents. (3) Improved phenotypic and genotypic diagnosis, treatment, and follow-up have accompanied the increased quantity and quality of health care in Iran over 50 years, despite economic sanctions. (4) The addition of 3 multidrug-resistant TB treatment centers in Shiraz, Mashhad, and Tabriz cities in southern, eastern and western Iran, respectively, now cover all regions of Iran. (5) Cooperation with the international community has improved, especially with the World Health Organization. (6) The activities of university centers in TB control have improved through skilled personnel training and improving education for the poor regions and suburbs. (7) Quality control programs for TB microbiology laboratories have been implemented. (8) The number of private medical diagnostic labs in the cities and the villages has grown substantially. (9) Finally, the Ministry of Health and Medical Education on TB program includes follow-up and continuous monitoring. According to the policy objective of the Ministry of Health in Iran, by the year 2025 , the detection rate of pulmonary TB will have increased to $>85 \%$ and the rate of treatment will have increased to $90 \%$.

\section{Acknowledgments.}

Financial support. No financial support was provided relevant to this article.

Conflicts of interest. All authors report no conflicts of interest relevant to this article.

\section{References}

1. Lohrasbi V, Talebi M, Bialvaei AZ, et al. Trends in the discovery of new drugs for Mycobacterium tuberculosis therapy with a glance at resistance. Tuberculosis 2018;109:17-27.

2. Punjabi CD, Perloff SR, Zuckerman JM. Preventing transmission of Mycobacterium tuberculosis in health care settings. Infect Dis Clin 2016;30:1013-1022.

3. World Health Organization. Global Tuberculosis Report 2018. Geneva, Switzerland: WHO; 2018.

4. Nasiri MJ, Dabiri H, Darban-Sarokhalil D, Rezadehbashi M, Zamani S. Prevalence of drug-resistant tuberculosis in Iran: systematic review and meta-analysis. Am J Infect Control 2014;42:1212-1218.

5. Department of Tuberculosis and Leprosy Control. Iran Ministry of Health and Medical Education website. http://tb-lep.behdasht.gov.ir/. Accessed February 5, 2019. 